\title{
Research on the Hydraulic Test Platform of Large Components
}

\author{
Yong-guang Liu \\ School of automation science and electric engineering \\ Beihang University \\ Beijng, China \\ lyg@buaa.edu.cn
}

\begin{abstract}
A hydraulic test platform of large component is designed in this paper. The technique method and design concept of test platform are studied. The structure diagram of the test platform is given, and the validity of the design by analyzing the structure of the loading system is also proved ,via UG software. The loading model is designed via the engineering simulation tools: AMESim and Simulink. In hydraulic test platform, coupling problem is hard to resolve. In this paper, new solution is proposed after the analysis for the coupling problem during cosimulation. Experimental results show that the system has good dynamic and static performance indices and excellent control results.
\end{abstract}

Keywords- electro hydraulic servo power control system, structural design, AMESim, Simulink

\section{INTRODUCTION}

The hydraulic test platform of large components has been widely used in various fields. Along with study of numbers of large and complex structure, mechanical experiment is a necessity before the delivery of production and use in many fields ,especially in the aerospace field, because there is a considerable difference between experimental research on the scale and full scale models .So hydraulic test platform is widely used nowadays. In hydraulic test platform, coupling problem is hard to resolve , which affects the accuracy of loading forces, so we need new hydraulic test platform for large components. We have finished a specific design scheme by structure design, hydraulic system design, control algorithm research, cosimulation and so on. This test platform can conduct loading static tests , and vertical force 10T, 20T are applied at three different points in two locations in this test . Fuzzy control algorithm make the loading force meet design accuracy. The test platform can also realize oblique $35^{\circ}$, amplitude $15 \mathrm{~T}$, frequency $1 \mathrm{~Hz}$ square wave loading.

\section{MEchanical StRUCtURE OF THE SyStem}

Mechanical structure is shown in Fig. 1. We accomplish mechanical structure design using UG software, slightly like the structure of gantry crane. The structure includes supporting pedestals, movable cross-beams, movable columns, 10T hydraulic cylinders, 20T hydraulic cylinders, tracks and so on.

\author{
Wang Cong \\ School of automation science and electric engineering \\ Beihang University \\ Beijng, China \\ xiaozwcong@163.com
}

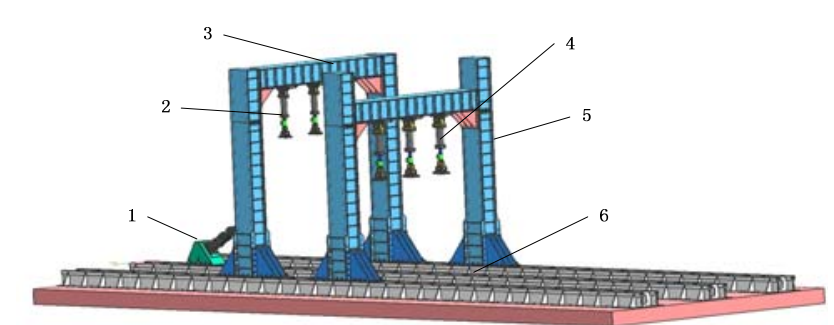

Note:1-supporting pedestal 2 -20T hydraulic cylinder 3 -movable crossbeam 4-10T hydraulic cylinder 5-column 6-track

Figure.1 Test platform loading structure diagram

Framework of the structure weighs about $12 \mathrm{~T}$, and the height is about $5.46 \mathrm{~m}$ and the length is $4.9 \mathrm{~m}$. The framework makes up of two parts, which could work independently. Each part can mount three hydraulic cylinders. For convenience in use and installation, the column is made up of two different pieces. Thanks to the holes in the columns, cross-beams could move along the columns. In order to resist external forces, we design a variety of stiffened plates at the junctions.

In order to guarantee that this system has enough precision, we study using finite-element analysis. Considering two parts of the framework are the same, we only need calculate one part which is working under the maximum load 20T.When framework satisfies request for utilization at that situation, the framework will work very well at other operating status. The calculation model for the bridge using finite element method (FEM) has been built, showed in Fig.2 (a). 10 nodes tetrahedron isoperimetric element is employed. Mesh size of finite elements is $30 \mathrm{~mm}$.At the junction of framework and track, fixed constraints are applied. The positions which hydraulic cylinders are installed on the framework are applied 20T forces, and weight of the framework should be considered.

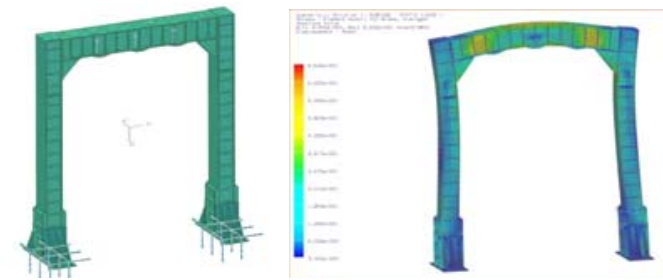

Figure 2（a）FEM calculation model (b) FEM computation results 
Fig.2(b) shows the computation results of FEM. It is showed that the maximum stress occurred in the middle of the cross-beam. Because the strength limit of Q235 is 500MPa, the factor of safety is about 7.5, meeting the requirement.

As required, we design a supporting pedestal, with 1000 meters long , 500 meters wide and 616 meters high(Fig.3). This structure weights about $320 \mathrm{~kg}$, which is fabricated with formed plates welded together. We use formed plates welding into cross for added strength inside.
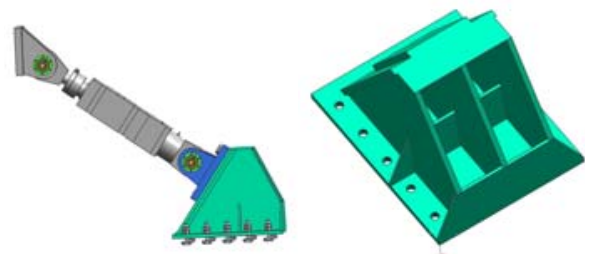

Figure. 3 The structure of the pedestal

Fig.6(a) show finite element model of the pedestal. In the present paper, 10 nodes tetrahedron isoparametric element is employed. Mesh size of finite elements is $30 \mathrm{~mm}$.Using one constrain that the undersurface is fixed, the static state and dynamic analysis applied to the frame of a few thickness plates with FEM modal analysis, distortion, stress, vibration and frequency are gained. Because in this situation, the pedestal is put dynamic loads on, 40T force is applied to the finite element models of the pedestal, to guarantee security. Then UG is used to analyze the stiffness and intensity of the structure, and the result shows that the mechanical structure is reasonable (Fig.4).

The result shows that the maximum displacement is located at the inclined plane, which is $0.04 \mathrm{~mm}$. The maximum stress is located in the bottom of the reinforcing plate, which is 20.25MPa. The intensity and rigid requirements of the pedestal are satisfied.

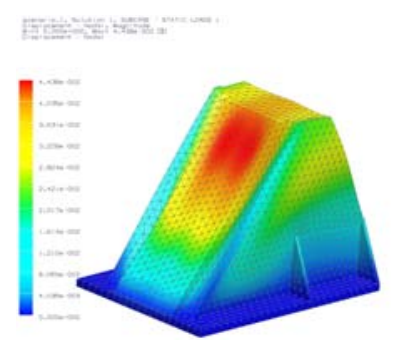

Figure.4 (a) Displacement of magnitude element（0.04mm)

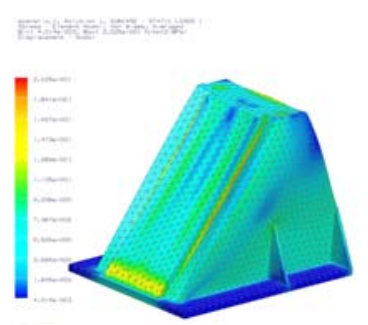

Figure.4（b ) Von Mises of element nodal（20.25MPa）

Because the structure could be loaded dynamically, dynamical modal analysis of the structure by use of UG

software is really necessary. The finite element model is the same as the model above.

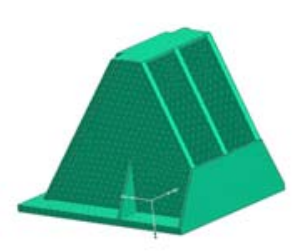

Figure 5（a） Finite element models of the pedestal

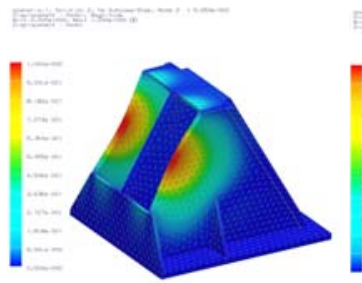

Figure.6 (a) The second order vibration and natural shape frequency $(502 \mathrm{~Hz})$ and natural shape frequency $(515 \mathrm{~Hz})$

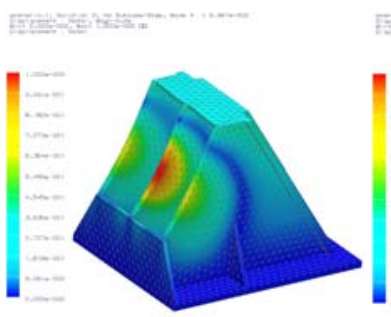

Figure.7 (a) The fourth order vibration and natural shape frequency $(606 \mathrm{~Hz})$

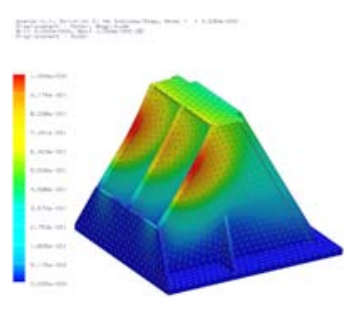

(b) The first order vibration and natural shape frequency $(407 \mathrm{~Hz})$

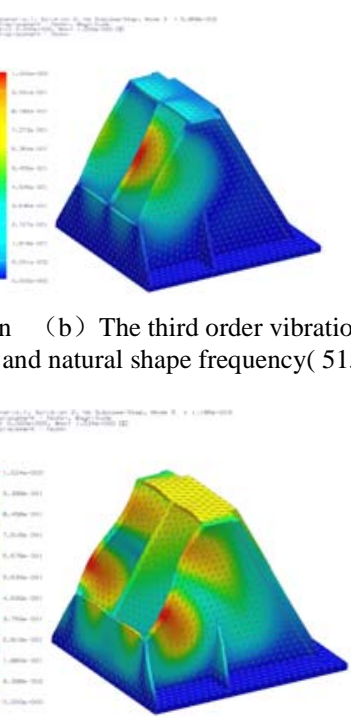

(b) The fifth order vibration and natural shape frequency $(797 \mathrm{~Hz})$
From Fig5(b) to Fig7(b), the models of the first five orders have been calculated by finite element method. We can see that the first order natural shape frequency is $407 \mathrm{~Hz}$, which is far more than that $10 \mathrm{~Hz}$ used in the experiment. This results suggest that stiffness of the pedestal meet the technical requirements.

\section{HYDRAULIC SYSTEM MODELING AND CO-SIMULATION}

In this paper, the loading model is designed. Based on simulation tools: Simulink and AMESim, we design the models for the two aspects of this system separately: this mechanical part and the hydraulic part. In hydraulic test platform, coupling problem is difficult to resolve. During the load process, different loads influence each other due to material deformation. In order to remove the impacts of loads, we should use different control algorithm.

In this paper, we take example of structural beams.When deformation of beams are small enough and the materials submit Hooke's Law, superposition method is applied to the calculation of deflection and rotation angle. Just like internal forces of beams, the deflection and rotation angle of beams are homogeneous linear differential equations of loads. In other words, superposition method is suitable for the deflection and rotation angle. When loads are applied to the beam at the same 
time, deflection and rotation angle in the cross-section of the beam equal to the algebraic sum of all deflection and rotation caused by each load.

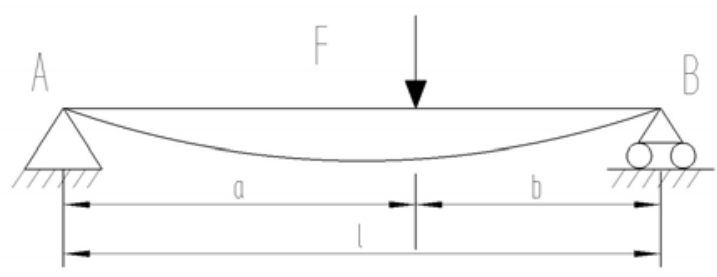

Figure.8 Simply supported beams

In Fig.8, deflection equation:

$$
\begin{aligned}
& v=-\frac{F b x}{6 E I l}\left(l^{2}-x^{2}-b^{2}\right), 0 \leq x \leq a \\
& v=-\frac{F b}{6 E I l}\left[\frac{l}{b}(x-a)^{3}-x^{3}+\left(l^{2}-b^{2}\right) x\right], a \leq x \leq l \\
& \text { Set }: \mathrm{a}=x_{1} 1, \quad x=x^{\prime} l, \mathrm{~b}=1
\end{aligned}
$$

When $0 \leq x \leq a$,

$$
\begin{aligned}
v & =-\frac{F\left(1-x_{1}\right) x}{6 E I l}\left[l^{2}-\left(x^{\prime} l\right)^{2}-\left(1-x_{1}\right)^{2} l^{2}\right] \\
& =-\frac{F\left(1-x_{1}\right) x^{\prime} l^{3}}{6 E I}\left(2 x_{1}-x^{2}-x_{1}^{2}\right)
\end{aligned}
$$

When $a \leq x \leq l$,

$$
\begin{aligned}
v & =-\frac{F\left(1-x_{1}\right) l}{6 E I l}\left\{\frac{l}{\left(1-x_{1}\right) l}\left(x^{\prime} l-x_{1} l\right)^{3}-\left(x^{\prime} l\right)^{3}+\left[l^{2}-\left(1-x_{1}\right)^{2} l^{2}\right] x_{1} l\right\} \\
& =-\frac{F\left(1-x^{\prime}\right) x_{1} l^{3}}{6 E I}\left(2 x^{\prime}-x_{1}^{2}-x^{\prime 2}\right)
\end{aligned}
$$

We considered that the beam is loaded at the two locations, shown in Fig.9 .

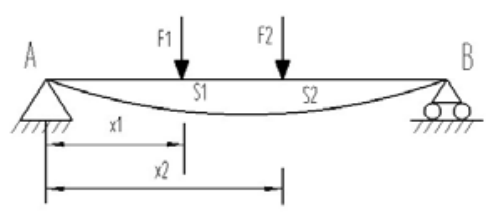

Figure.9 Deflection analysis of the beam

$F_{1}$ at the location 1:

$$
S_{1 F_{1}}=-\frac{F_{1}\left(x_{1}-1\right) x_{1} l^{3}}{6 E I}\left(2 x_{1}^{2}-2 x_{1}\right)
$$

$F_{1}$ at the location 2:

$$
S_{2 F_{1}}=-\frac{F_{1}\left(x_{2}-1\right) x_{1} l^{3}}{6 E I}\left(x_{1}^{2}+x_{2}^{2}-2 x_{2}\right)
$$

$F_{2}$ at the location 1:

$$
S_{1 F_{2}}=-\frac{F_{2}\left(x_{2}-1\right) x_{1} l^{3}}{6 E I}\left(x_{1}^{2}+x_{2}^{2}-2 x_{2}\right)
$$

$F_{2}$ at the location 2:

$$
S_{2 F_{2}}=-\frac{F_{2}\left(x_{2}-1\right) x_{2} l^{3}}{6 E I}\left(2 x_{2}^{2}-2 x_{2}\right)
$$

deflection of the cross-section 1:

$$
S_{1}=S_{1 F_{2}}+S_{1 F_{1}}
$$

deflection of the cross-section 2:

$$
S_{2}=S_{2 F_{2}}+S_{2 F_{1}}
$$

Set: $a_{11}=-\frac{\left(x_{1}-1\right) x_{1} l^{3}}{6 E I}\left(2 x_{1}^{2}-2 x_{1}\right)$

$$
\begin{aligned}
& a_{12}=a_{21}=-\frac{\left(x_{2}-1\right) x_{1} l^{3}}{6 E I}\left(x_{1}^{2}+x_{2}^{2}-2 x_{2}\right) \\
& a_{22}=-\frac{\left(x_{2}-1\right) x_{2} l^{3}}{6 E I}\left(2 x_{2}^{2}-2 x_{2}\right)
\end{aligned}
$$

Thus $\left\{S_{1}=a_{11} F_{1}+a_{12} F_{2}\right.$

$$
\left\{S_{2}=a_{21} F_{1}+a_{22} F_{2}\right.
$$

Set: $\quad S=\left\{\begin{array}{l}S_{1} \\ S_{2}\end{array}\right\}, A=\left\{\begin{array}{l}a_{11}, a_{12} \\ a_{21}, a_{22}\end{array}\right\}, F=\left\{\begin{array}{l}F_{1} \\ F_{2}\end{array}\right\}$

Thus $S=A F$

$$
F=A^{-1} S
$$

$$
A^{-1}=\left\{\begin{array}{l}
\frac{a_{22}}{a_{11} a_{22}-a_{12}{ }^{2}}, \frac{-a_{12}}{a_{11} a_{22}-a_{12}{ }^{2}} \\
\frac{-a_{12}}{a_{11} a_{22}-a_{12}{ }^{2}}, \frac{a_{11}}{a_{11} a_{22}-a_{12}{ }^{2}}
\end{array}\right\}
$$

Where, A and $A^{-1}$ have relationships with features of the beam and show no effect on external forces.

In this paper, we consider $F_{1}$ is applied at the location $\frac{1}{3} l$ and $F_{2}$ is applied at the location $\frac{2}{3} l$ as a special case.

Hence, we obtain

$$
A^{-1}=\left\{\begin{array}{l}
\frac{8}{11}, \frac{-567}{80} \\
\frac{-567}{80}, \frac{8}{11}
\end{array}\right\}
$$

According to $F=A^{-1} S$, simulink model can be obtained, shown in Fig.10.

The model of hydraulic system can be built via software AMESim (Fig.11) according to hydraulic plans. During the cosimulation, the subroutine for loads, which is written in simulink, will be exported to AMESim as a sub-model by invoking the interface of these tools. To improve the accuracy of simulation, the co-simulation's model particularly takes the detailed characters of real machine into account. 


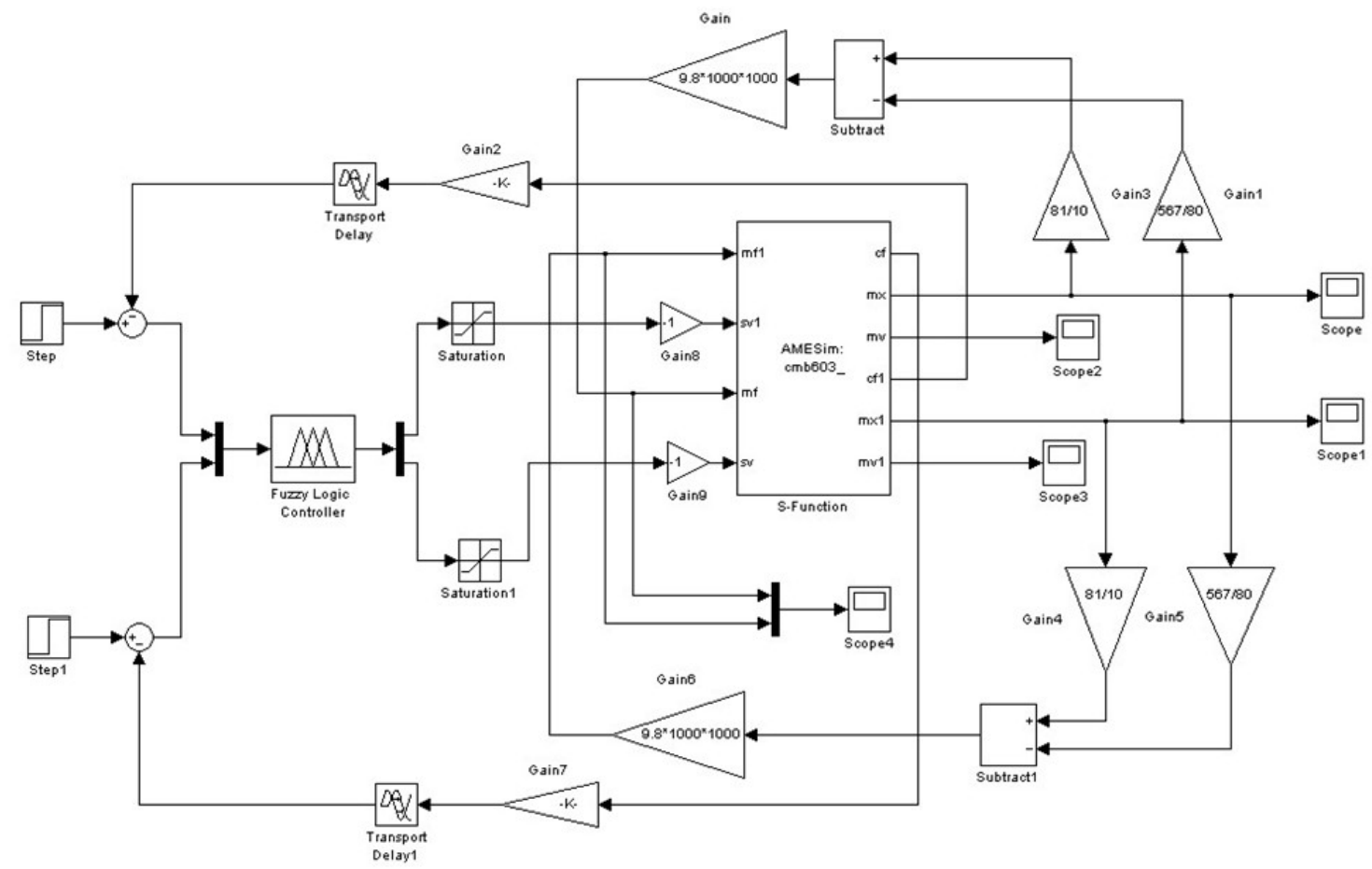

Figure.10 Simulink model in the co-simulation(simulink)

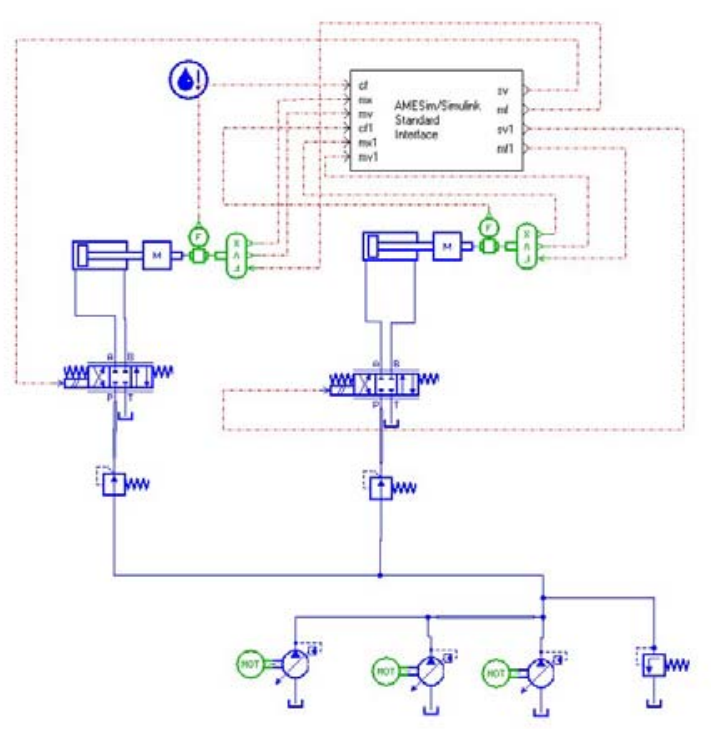

Figure.11 Hydraulic model in the co-simulation(AMESim)

Because two forces are applied to this system, the PID control algorithm could hardly provide precise force control duo to the coupling problem. In order to get precise force control, fuzzy algorithm is used in this system, and the design of the controller uses the MATLAB fuzzy toolbox. The errors of output forces are used as control signal of fuzzy algorithm, which decide the output signal of fuzzy toolbox. When the forces are $10 \mathrm{~T} 、 10 \mathrm{~T}$ or $20 \mathrm{~T} 、 20 \mathrm{~T}$, experiments are presented and this control strategy gives good performance, shown in Fig.12 and Fig.13.

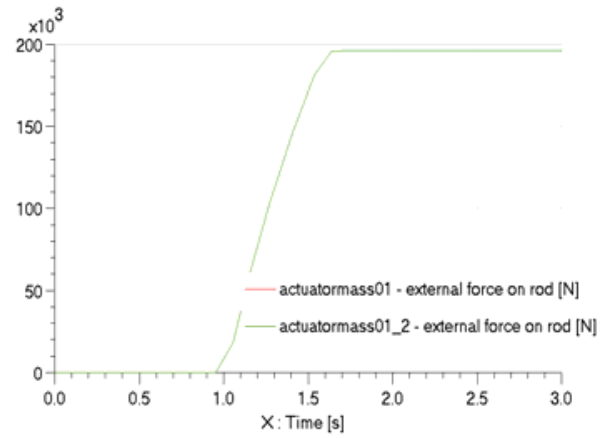

Figure.12 Export force diagram (10T,10T)

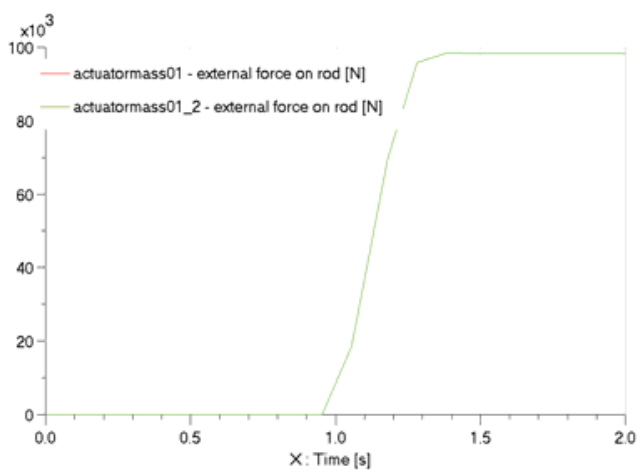

Figure.13 Export force diagram (20T,20T) 


\section{CONCLUSION}

In design of the test platform, three-dimensional modeling is done and the structure of the test platform is analyzed using UG in order to ensure that test platform has enough strength and stiffness. For solving the difficult coupling problem of loading forces, fuzzy control is used in the system. Then, simulation results of co-simulation of AMESim and Simulink demonstrate better performance of this neural fuzzy controller, which conform that our control policy can settle the coupling problem of loading force. Experimental results show that the system has good dynamic and static performance indices and excellent control results, and also this test platform has important theoretical and practical significance in future.

\section{REFERENCES}

[1] Zhou limin. Research On Electro-hydraulic Servo Loading System Applied On Large Columnar Component Fire Resistance Test Equipment [D]. Hangzhou: Zhejiang University,2008.

[2] Liang Laiyu, Li Weijia. The Design of Hydraulics System For Multicomponent Force Loading Test-platform[M],CHINESE HYDRAULIC\&PNEUMATICS,2011(1).

[3] Lei Tianjue. Hydraulic Engineering Manual[M].Beijing:China Machine Press, 1990.

[4] Zhang Er. Structural Design Analysis of The Oversize Multifunctional Structure Test Loading System[D]. Beijing:Beijing Jiaotong University,2005.

[5] Fu Yongling, Qi Xiaoye. AMESim system modeling and simulation $[\mathrm{M}]$. Beijng: Beijing University of Aeronautics and Astronautics Press, 2006.

[6] Merritt.H.E. Hydraulic Control System [M], 1967. 